\title{
Blockchain Study and Analysis with a View to Optimizing Security on the Aspects of Land Registry
}

\author{
Georges Bell Bitjoka ${ }^{1}$, Sebastian Yves Wono Emvudu ${ }^{2}$, Basile Bete Mbezele ${ }^{2}$ \\ ${ }^{1}$ Departement de Telecommunications, National Advanced School of Engineering, University of YaoundéI, Yaoundé, Cameroon \\ ${ }^{2}$ Department of Computer Science, Faculty of Sciences, University of YaoundéI, Yaoundé, Cameroon
}

\section{Email address:}

georges@bellbitjoka.com (G. B. Bitjoka), yves.emvudu@minsup.gov.cm (S. Y. W. Emvudu), mbezelebete@gmail.com (B. B. Mbezele)

\section{To cite this article:}

Georges Bell Bitjoka, Sebastian Yves Wono Emvudu, Basile Bete Mbezele. Blockchain Study and Analysis with a View to Optimizing Security on the Aspects of Land Registry. American Journal of Computer Science and Technology. Vol. 3, No. 2, 2020, pp. 27-37. doi: $10.11648 /$ j.ajcst.20200302.12

Received: April 13, 2020; Accepted: May 5, 2020; Published: June 4, 2020

\begin{abstract}
Land, according to most governments, is an important asset in development. All over the world, it expresses family cohesion to a certain extent, and presents itself as a basis for the development of any human project. Thus, the regulation of its use and occupation is required. In Cameroonian legislation, the land system is using a special book called "land Book". This book represents a document used by a registrar to record users 'land titles, in order to prove the authenticity, availability, and confidentiality of its holder. To date, its land system still suffers from several problems, in particular: the dilapidated state and disappearance of land books, the existence of several geodetic systems, disappearance of leaflet, sale of false land titles, double sales of land titles, and attempts to expropriate a person or a community by the administration or by third parties. Today, most of the cases pending in the courts are land disputes, and since then they have continued to increase due to demographic pressure, and the rate of urbanization in Cameroon. The objective of this paper is to solve these problems by designing a Framework via a particular type of blockchain approach in order to improve the security and scalability of the land register in Cameroon.
\end{abstract}

Keywords: Land Register, Land Book, Framework, Blockchain, Land Title, Leaflet/Folio

\section{Introduction}

Since the creation of the national conservation of land registers of users in Cameroon, the staff in charge of this conservation and users faces daily many problems, due to: the dilapidated state of land registers, voluntary or involuntary disappearance of land registers and leaflet, sale of false titles, double sale, presence of several geodesic networks, and many others. Indeed, since the enthronement of land titles in Cameroon (1974) these registers are subject to various manipulation due to transactions (parceling, transfer, sale,...), we also have the manipulation of these documents by several agents ( conservation secretariat) thus increasing the risk of errors, moreover it very often happens that one does not know who holds the register at a given time. According to numerous reports on the question of land, in particular that of The African Development Bank Group (AfDB) at the request of the Cameroonian government in 2009 on "THE DIAGNOSTIC STUDY FOR THE
MODERNIZATION OF THE SECTORS OF THE CADASTRE AND AREAS" ${ }^{1}$, another report from the African Development Fund (ADF) still at the request of the Cameroonian government on the "SUPPORT PROJECT FOR MODERNIZING THE CADASTRE AND BUSINESS CLIMATE (PAMOCCA)" ${ }^{2}$, shows that several factors are increasing the problems of land in Cameroon in particular:

Population growth: The population is experiencing an annual growth rate of around $2.9 \%$, which will ultimately generate strong pressure on land resources with the risk of increasing land conflicts. This pressure is fueling growing conflicts, corruption and the exclusion of the poor from

\footnotetext{
1 https://www.afdb.org/fileadmin/uploads/afdb/Documents/Project-andOperations/Cameroon $\% 20$ -

\%20Diagnostic $\% 20$ Study $\% 20$ for $\% 20$ Modernization $\% 20$ of $\% 20$ the $\% 20$ Lands $\% 20$ and $\% 20$ Surveys $\% 20$ Sectors $\% 20$ FR.pdf.

2 https://www.afdb.org/fileadmin/uploads/afdb/Documents/Project-andOperations/CAM_PAMOCCA_Rapport_d_\%C3\%A9valuation_30sept2010_Final_full_sans_annexes_Approuv\%C3\%A9_Final2_.pdf.
} 
access to property. The numerous cancellations of land titles observed in recent years are proof of a form of land insecurity detrimental to the private sector, which is nonetheless recognized as one of the engines of growth.

The rate of urbanization: The rate of urbanization is estimated at $45 \%$ with an increase of 6 to $6.5 \%$ per year. This indicator, which reveals a strong migratory trend towards urban areas, indicates that more and more, the pressure on land will accelerate in urban centers, for housing needs, socio-collective equipment and services. Problems of land management, securing rights and transactions will increase in urban and semi-urban centers. The formal land market will tend to be concentrated in urban and peri-urban areas. More than $50 \%$ of those who registered land in the cadaster worked in the civil service and only $5 \%$ were peasants (World Bank Report).

\section{Objective}

From the above introduction, how do you secure the Land Registry, so that users can access their property transparently while improving scalability? The aim of this paper is to implement blockchain-based framework.

So ta answer the question we are going to:

Study the keys concepts of blockchain, with the aim of to identify the type of blockchain.

Study the Hyperledger Fabric and propose a framework to solve the problem.

Analyze the scalability of Hyperledger Fabric.

\section{Literature Review}

\subsection{Internal Review: Case of Cameroon}

In Cameroon, being the owner of a plot of land means having the land title linked to this plot according to Decree No. 76-165 of April 27, 1976. Obtaining the land title goes through the registration procedure to the Minister of Domains, Cadastral and Land Affairs.

The registration procedure is a way of obtaining land title. For this, it must be distinguished from derived modes: It is first of all the fragmentation land which consists in having a title on a plot of land which has previously been the subject of a first land title; and the transfer, which is the fact of having the same titled land but of changing ownership; we can also mention the donation or cession which consists for the holder of a land title to cede his rights on the land to a third party; Finally, the merger which consists of bringing together two or more land titles to obtain a single one when said land is located side by side.

Once the procedure for obtaining the land title has been approved, the securing of the land title begins, through the manipulation of the following Registers: opponents' registers, deposit registers, and finally the land Register.

In its decree $n^{\circ} 2012 / 390$ of September 18, 2012 on the organization of the Ministry, in article 106: placing under the authority of a land registrar, registration of land, registration of all real rights in the Land Registry, the recording of real estate rights in the Land Registry, the keeping and conservation of the Land Registry and title files. Figure 1. below, explain this functioning.

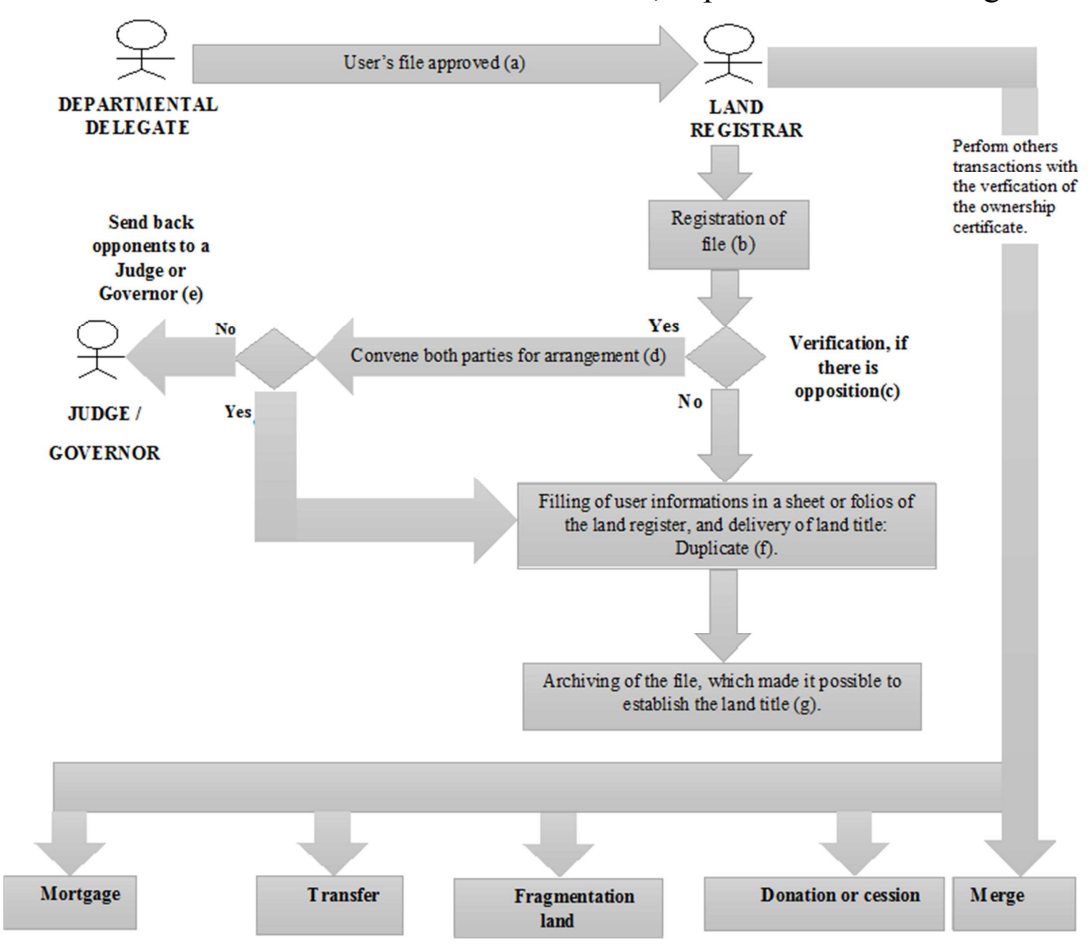

Figure 1. Workflow of transaction in land registry office.

(a) Basic security elements (user file approved): Depending on the legal status of an area requested by a potential buyer, there is a registration procedure accompanied by a land title file. A file approved by the Departmental 
Delegate supposes to contain all the elements (technical file with the information of demarcation by a sworn surveyor, and a stamped request).

(b) Registration of file in the deposit Register: after phase (a), an approved file is sent to the land registrar who registers it, if it is not already registered, in order to be able to trace the dossier in its registration process by assigning it a reference number. This trace also makes it possible to know if a user has a false title or not. E.g.: consider an analytical slip to which a deposit number, its corresponding volume and the name of the user are carried, it can therefore happen that the name does not correspond in the deposit register: thus to find out, the land registrar always proceeds to verification to filter forged files. (Interview by Land Registrar C of Mfoundi Department).

(c) Check, if there is opposition: In the direct registration process, well before the user's file arrives at the Land Registrar's office for property registration, the regional chief of land affairs checks the regularity of the file and affixes the regulatory visa, prepares publication of the boundary closure notice in the regional bulletin of land and property notices.

(d) If opposition occurs: then the land registrar will convene both parties for arrangement. If not, then send back opponents to a Judge or Governor.

(e) Send back opponents to a Judge or Governor: If there is opposition, the land registrar convenes the both parties for an arrangement, in the event that there has been an arrangement, the land registrar enters the rights in the land register, issues a copy of the land title (duplicate) to the individual applicant or to the head of the community of applicants, followed by the sending of the merits of the file to the archives for classification.

\section{Problems}

Dilapidated state of the

old Land registers.

Office fire.

\section{Verification of the authentication of a Land Title.}

Loss of a Land Title

Disappearance of the sheets (folios).

Sale of false Land Titles or double sale.

Disappearance of the Land Register, Register of deposits, and file. land registry office's solutions Transcription.

Restore the Land Title with the user's copy (duplicate).

Establishment of property certificates.

Procedure for establishing a Duplicate.

Authorization of judge to restore lost sheet.

identify the

Perpetrators of this criminal act and to bring them to justice.

Restore the land register with the help of the judge, and users in possession of their duplicates. (f) Filling of user information in a sheet of the Land Register: If there has been no opposition, the land registrar enters your rights in the land register on a sheet or folio to mention therein the land title information. Each sheet has a serial number, i.e. if the first title has the number 1 , the second title has the number 2 and so on. The land register therefore has no right to omit or skip a sheet, to make a sheet disappear voluntarily or not; if this situation occurs, the judge's authorization will be required to restore the lost slip, this approach aims to avoid fraud, tampering and other facts that aim to compromise the process of establishing titles and securing the properties of users. Finally Issue a copy of the land title (duplicate) to the individual applicant or to the head of the community of applicants.

(g) Archiving of the file: after the registration of the rights in the land register, it sends the physical file which made it possible to establish the following title in the archives for classification.

(h) Verification of the certificate of ownership: Land title is an asset, therefore is often the subject of various manipulations: mortgage, transfer, assignment, and sale. To carry out a transaction on a land title, the curator checks whether it is not the subject of a transaction in progress.

\subsection{Security Analysis of Land System in Cameroon and Discussion}

Land management with this system, is encountering enormous difficulties. According to Land registrar $\mathrm{C}$ of the Mfoundi department, the solutions to these difficulties are summarized in the table below:

\section{Weaknesses}

Transcribe the information from the old land registers into the new land registers in order to standardize the information. If a fire happens to occur at the land registry office level, then the Land registrar would use the copies of users' Land Titles to restore the Land Titles from the Land Register. This does not always guarantee the reliability of the solution, because if the user had a fake, we will then assign an authentic title to a fake duplicate.

A land title is very often the subject of transactions: mortgage, sale, transfer, and many others. To verify this, simply establish a certificate of ownership to prove that a Land Title is free from all transactions at a given time.

If a user has lost his Land Title, there is a procedure for establishing a Duplicate.

The folio represents the sheet dedicated to the authentic information of a Land Title; if it disappears, we could take advantage to tamper with, tamper with the user's Land Title.

The sale of fake Land Titles.

Restore the Land Title with the user's copy, or restore the Land Register with the users' copies. In short, the Curator uses his intuition and the judge's help to establish the Land Titles by all the technical means in his possession. 


\subsection{External Review: Ghana, Honduras, India}

Land, according to most governments, is an important asset in development; as such its regulation is essential. Some countries in the world have already digitized their land systems, in order to improve reliability in their management: others with or without the blockchain. We will focus on three (03) main works related to similar problems that they address in a particular context. Namely, the Bitland ${ }^{3}$ project, EPIGRAPH in Honduras ${ }^{4}$, and finally the framework design project using the Blockchain [3].

In Ghana, the mission of the Bitland project consists in recording the rights of ownership and use of land and real estate in a secure electronic format. In order to make them accessible to all, according to the founder of the project Narigamba Mwinsuubo noted that in his country of origin (Ghana) the land system is a real headache. Unregistered land, corruption and bureaucracy are obstacles to everything local or foreign investment in the real estate sector in Ghana. Clarifying property rights will not only reduce corruption, but also mobilize billions of dollars in locked-in capital, since land that has no land title cannot be used as assets. Nowadays this project has not stopped offering new services: BITLAND has chosen to launch its own encryption token based on Bitshares Graphene 2.0 technology under MIT license.

Honduras was said at the time to be particularly interested in the Blockchain as a solution to overcome a struggle with land title fraud, where land ownership records were so badly open to corruption that bureaucrats could access them, change them and give themselves legal title to beachfront property. According to a statement made by Factom Chief Executive Officer Peter Kirby, the deal has officially stalled, with the working prototype they had been working on simply not happening, although Kirby remains surprisingly confident that it may happen in 2016.

Finally, the work on which we will rely, is "the implementation of Entangled Blockchains system in Land Registry Management" Because to solve the problem, they used a scientific approach, and at the end of paper, presented the limits [1]. India is one of the countries, has understood the merits of land regulation by setting up automatic systems. Her desire to tackle the land problem is illustrated with the list of systems she has already experienced: National Land Records Modernization Program (NLRMP), Electronic Citizen Services (ECS), JAN SEVA KENDRA, National eGovernance Plan (NeGP), Computerization of Registry Department (CORD), etc.

On this paper, the problem they have faced is the coordination between the various departments, and multiplicity of land records, via entanglement of blockchains. The tool used to implement the system is a Hyperledger Fabric. Concerning the limits of the paper we have: they

3 https://www.cryptokemet.com/ghana-bitland-cadastre-blockchain/, consulted on 29/02/2020.

4 https://siliconangle.com/2015/12/27/factoms-blockchain-land-reigstry-tool-trialstalls-due-to-the-politics-of-honduras/, consulted on 29/02/2020. have not done optimization in terms of space, which is one of the major factors in terms of the scaling of blockchains. On this paper, we are going to ameliorate scalability.

\section{Research Methodology}

In this paper, we're going to present a framework used to improve the security and scalability of land register in Cameroon. We used different tools and technics to reach it, these include:

Study keys concepts of blockchain not only to present components used to ameliorate the security of Land Register, but to identify the type of blockchain used to build the framework.

Analysis of the Hyperledger scalability, so that to propose the improvement of scalability of Hyperledger.

\subsection{Blockchain}

Blockchain is a technology for storing and exchanging information that is secure, transparent and works without the need of a centralized trusted authority. Many experts agree that it is like a great book to write about, but not to erase or destroy. According to mathematician Jean-Paul Delahaye ${ }^{5}$. The blockchain is "a very large notebook that, freely and free of charge, everyone [can] read, on which everyone [can] write, but which [is] impossible to erase and indestructible". Resources can be tangible (e.g., money, houses, cars, lands) or intangible (e.g. copyrights, digital documents, and intellectual property rights). In general, anything that has a value can be tracked on a blockchain network to reduce its security risks and save the cost of security monitoring for all involved (Tara Salman, and Maede Zolanvari, 2018).

\subsubsection{Blockchain Architecture}

Blockchain is a sequence of blocks, which holds a complete list of transaction records like conventional public ledger (Lee Kuo, 2015).

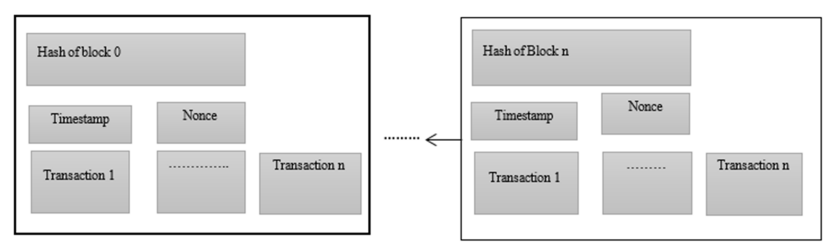

Figure 2. Block structure (Zibin Zheng, 2017).

Figure 2 is an example of blockchain. A previous block hash contained in the block header, a block has only one parent block. It is worth noting that uncle blocks (children of the block's ancestors) hashes would also be stored in ethereum blockchain [5]. The first block of a blockchain is called genesis block which has no parent block. We then explain the internals of blockchain in details. The following figure 3 present it.

5 https://www.argusdelassurance.com/tech/comment-ca-marche-lablockchain. 155554 . 


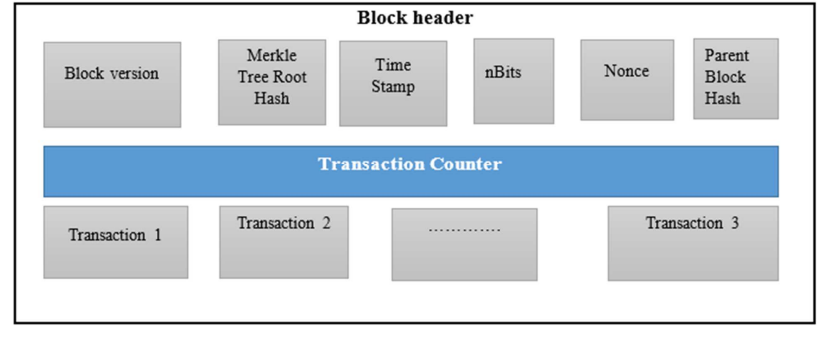

Figure 3. Block structure.

This include

Block: has two parts block header and the block body as shown in Figure 3. The block header includes:

Block version: indicates which set of block validation rules to follow.

Merkle tree root hash: the hash value of all the transactions in the block.

nBits: target threshold of a valid block hash.

Timestamp: current time as seconds.

Nonce: a 4-byte field, which usually starts with 0 and increases for every hash calculation.

Parent block hash: a 256-bit hash value, which points to the previous block.

Digital Signature: Each user owns a pair of private key and public key. The private key is used to sign the transactions. The digital signed transactions are spread throughout the whole network and then are accessed by public keys, which are visible to everyone in the network. The typical digital signature algorithms used in blockchain include elliptic curve digital signature algorithm (ECDSA) (Johnson et al., 2001).

\subsubsection{Key Characteristics of Blockchain}

Immutable: Once the information and exchanges have been registered in a Blockchain, they cannot be modified and are permanently stored there.

Transparent: There is no "right of entry". Anyone can consult all the exchanges and transactions recorded on a blockchain, from the first block created. Once a document is registered on the blockchain, this is enough to prove that the latter exists at time $\mathrm{T}$ and that it has not been modified. The blockchain is qualified as transparent because everyone can download it in its entirety and check its honesty at any time ${ }^{6}$. All blockchain users can thus view current and past transactions ${ }^{7}$.

Secure: Decentralized hosting also makes blockchain a secure technology: it makes it almost impossible to delete all copies of documents, which exist on a multitude of servers around the world. The blockchain has great resistance, because all the data is copied to the different servers. This makes it resistant to cyber-attacks or state control. Indeed, if it is possible to attack one or more computers, it is more complicated to attack the blocks of information copied in all

6 Claire Fénéron Plisson, « La blockchain, un bouleversement économique, juridique voire sociétal », I2D - Information, données \&documents, (Volume 54), p. 20-22, mars 2017.

7Thomas Dupont, op.cit. the computers connected to the network. This provides the blockchain with a high level of security. The blockchain is therefore considered unassailable and inviolable ${ }^{8}$. However, this also makes it difficult to adjust.

Decentralization: In conventional centralized transaction systems, each transaction needs to be validated through the central trusted agency (e.g., the central bank), inevitably resulting to the cost and the performance bottlenecks at the central servers. Contrast to the centralized mode, third party is no longer needed in blockchain. Consensus algorithms in blockchain are used to maintain data consistency in distributed network.

Transparent: The data's record by blockchain system is transparent to each node, it also transparent on update the data that is why blockchain can be trusted.

\subsubsection{Type of Blockchain}

Blockchain technologies can be roughly divided into three types. These include: Public blockchain, Consortium blockchain, and Private blockchain.

Public blockchain: Everyone can check the transaction and verify it, and can also participate to the process of getting consensus. Like Bitcoin and Ethereum are both public Blockchain. Figure 4 shows public blockchain.

Consortium blockchains: In the consortium blockchain there is an administrative node, which is selected by the network participants initially based on the optimal ways to achieve their business goals, for example, in the case of a partnership. Data in such networks can be public and private (for example, confidential information), so the network itself can be regarded as partially decentralized. An example of such a network is the Hyperledger platform. Figure 5 shows consortium blockchain. [12].

Private blockchain: Node will be restricted, not every node can participate this blockchain, has strict authority management on data access. Figure 6 shows private blockchain.

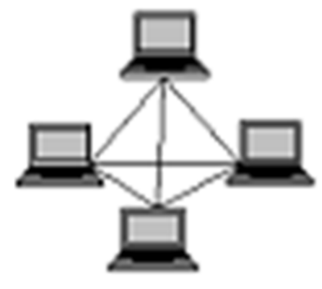

Figure 4. Public blockchain

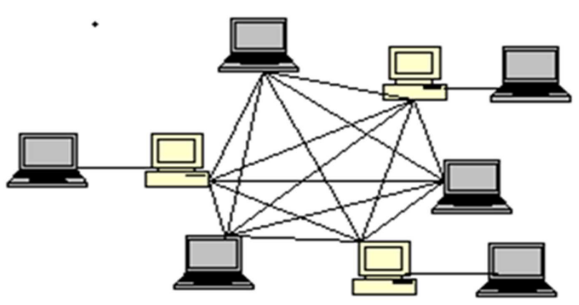

Figure 5. Consortium blockchain. 


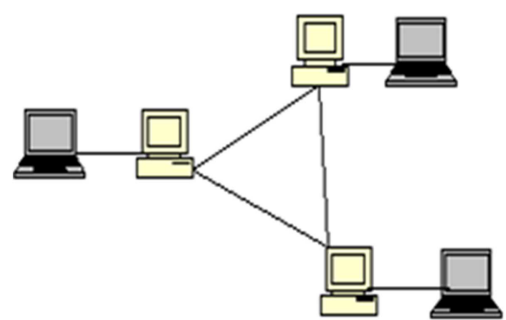

Figure 6. Private blockchain.

Tableau 1 type of blockchains (Iuon-Chang Lin, 2017).

\subsubsection{Consensus Mechanism}

Consensus function is a mechanism that make all blockchain nodes have agreement in same message, can make sure the latest block have been added to the chain correctly, guarantee the message that stored by node was the same one and won't happened "fork attack", even can protect from malicious attacks.

In blockchain, how to reach consensus among the untrustworthy nodes is a transformation of the Byzantine Generals (BG) Problem, which was raised in [6]. In BG problem, a group of generals who command a portion of Byzantine army circle the city. Some generals prefer to attack while other generals prefer to retreat. However, the attack would fail if only part of the generals attack the city. Thus, they have to reach an agreement to attack or retreat. How to reach a consensus in distributed environment is a challenge. It is also a challenge for blockchain as the blockchain network is distributed. In blockchain, there is no central node that ensures ledgers on distributed nodes are all the same. Some protocols are needed to ensure ledgers in different nodes are consistent. We next present several common approaches to reach a consensus in blockchain.

PoW (Proof of work): is a consensus strategy used in the Bitcoin network [7]. In a decentralized network, someone has to be selected to record the transactions. The easiest way is random selection. However, random selection is vulnerable to attacks. So if a node wants to publish a block of transactions, a lot of work has to be done to prove that the node is not likely to attack the network. Generally the work means computer calculations.
In PoW, each node of the network is calculating a hash value of the block header. The block header contains a nonce and miners would change the nonce frequently to get different hash values. The consensus requires that the calculated value must be equal to or smaller than a certain given value. When one node reaches the target value, it would broadcast the block to other nodes and all other nodes must mutually confirm the correctness of the hash value. If the block is validated, other miners would append this new block to their own blockchains. Nodes that calculate the hash values are called miners and the PoW procedure is called mining in Bitcoin.

PoS (Proof of stake), is an energy-saving alternative to PoW. Miners in PoS have to prove the ownership of the amount of currency. It is believed that people with more currencies would be less likely to attack the network. The selection based on account balance is quite unfair because the single richest person is bound to be dominant in the network. As a result, many solutions are proposed with the combination of the stake size to decide which one to forge the next block. In particular, Blackcoin [8] uses randomization to predict the next generator. It uses a formula that looks for the lowest hash value in combination with the size of the stake. Peercoin [9] favors coin age based selection. In Peercoin, older and larger sets of coins have a greater probability of mining the next block.

PBFT (Practical byzantine fault tolerance) is a replication algorithm to tolerate byzantine faults [10]. Hyperledger Fabric [11] utilizes the PBFT as its consensus algorithm since PBFT could handle up to $1 / 3$ malicious byzantine replicas. A new block is determined in a round. In each round, a primary would be selected according to some rules. And it is responsible for ordering the transaction. The whole process could be divided into three phase: pre-prepared, prepared and commit. In each phase, a node would enter next phase if it has received votes from over $2 / 3$ of all nodes. So PBFT requires that every node is known to the network.

\subsubsection{Functioning of Blockchain}

According to figure 7 below, the main working processes of blockchain are as follows:

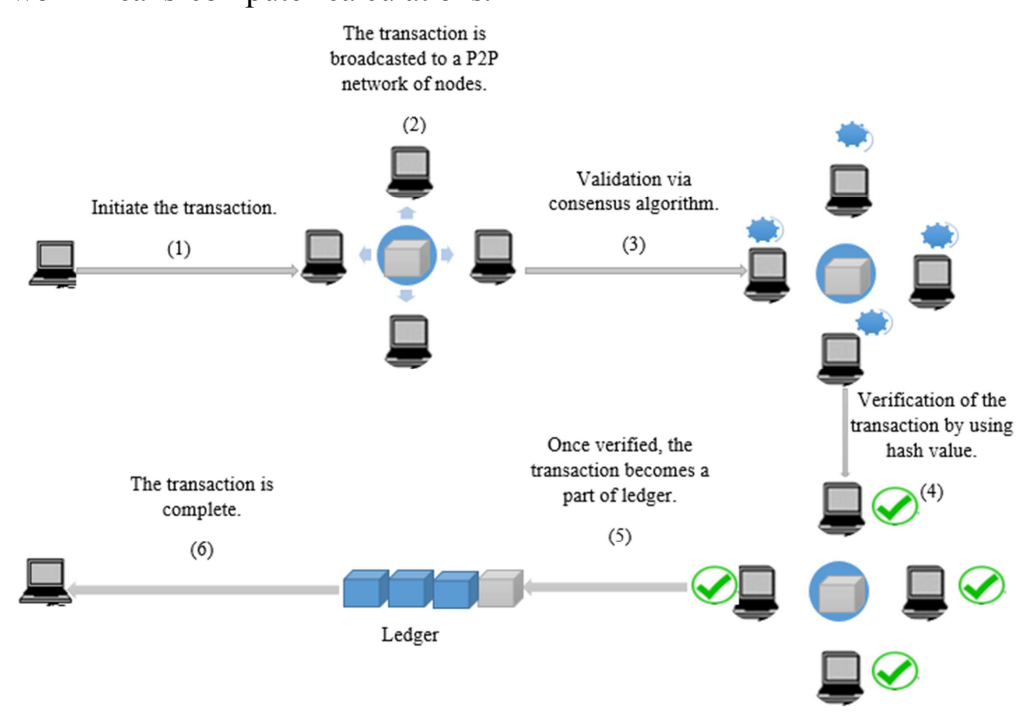

Figure 7. Functioning of blockchain. 
Step 1: a node requests a transaction.

Step 2: The transaction is broadcasted on a public P2P network (Blockchain network) composed of several nodes.

Step 3: The network of nodes (miners) validates the transaction using hashing algorithms such as: Proof of work (PoW), proof of stake (PoS), or PBFT (Practical Byzantine Fault Tolerance)...

Step 4: Once verified, the transaction is combined with other transactions to create a new block of data for the general ledger.

Step 5: The new block is added to the existing blockchain in a form that is permanent and unalterable.

Step 6: Finally the transaction will be completed successfully.

\subsection{Choice of the Blockchain Type [12]}

The choice of a particular type of blockchain depends of the system functioning. For example, for small businesses where accounting is maintained by a separate institution, a suitable option would be to use a private blockchain network in which a distributed registry would become a single source of truth.

In the case of Land Register, where the user wants to access the land title, a consortium network will approach. Land Register would be available to read by all stakeholders, but only authorized nodes like: Land Registrar, the land title, manipulation of land title (donation or cession, fragmentation land, merger, transfer, etc.) may record it.

Hyperledger Fabric is an example of consortium blockchain.

\subsection{Framework to Improve Security and Scalability of Land Book with the Hyperledger Fabric}

As mentioned above, each country has its legislation, unlike India, in Cameroon there is a single structure that manages land: the Ministry of Domains, Cadaster and Land Affairs (MDCLA). In this Ministry, only the Land Registrar is ability to establish a land title, including other operations that can be carried out on a land title (transfer, fragmentation, sale, mortgage, transfer, etc.). In the following, we will therefore define the nodes which will verify the transactions including those which will validate the transactions, and finally present some approaches which make it possible to solve the problem of "scalability".

\subsubsection{Hyperledger Fabric}

Instead of The order-execute architecture used by public or permissionless blockchain, Fabric introduces a new blockchain architecture aiming at resiliency, flexibility, scalability, and confidentiality, this architecture is called: execute-order-validate. Order-execute architecture suffers from many limitations [14]: there is no one-size-fits-all (BFT) consensus protocol, trust model of transaction validation is determined by the consensus protocol and cannot be adapted to the requirements of the smart contract, Smart contracts must be written in a field, non-standard, ordomain-specific language, which hinders wide-spread adoption and may lead to programming errors, while in Fabric architecture Fabric is the first blockchain system to support the execution of distributed applications written in standard programming languages, in a way that allows them to be executed consistently across many nodes. It separates the transaction flow into three steps, which may be run on different entities in the system:(1) executing a transaction and checking its correctness, thereby endorsing it (corresponding to "transaction validation" in other blockchains); (2) ordering through a consensus protocol, irrespective of transaction semantics; and (3) transaction validation per application specific trust assumptions, which also prevents race conditions due to concurrency.

\subsubsection{Fabric Components}

The figure 8 below, presents components used in the Hyperledger Fabric:

Peers: We know that blockchain is nothing but a distributed network of nodes (in this case peers) which makes the peers the building blocks of a blockchain network. In fabric a peer by definition can host multiple instances of ledgers and smart contracts (defined later). Peers are classified into three categories which are given below:

Endorsing Peers: Receive transaction proposals from the client application for endorsements. Smart contracts must be present to execute the proposal.

Committing Peers: Commits the transactions and maintain the state of the ledger.

Ordering Peers: Special type of peers where the endorsed transactions are received ordered into blocks and are distributed to validating peers for validation and the updating of their respective ledgers.

Ledgers: The term ledger in Hyperledger fabric is associated with two distinct parts - a State Database and transaction log. The state DB records the current values of assets and makes it easy for the developer to access the values instead of traversing the entire chain. Fabric allows the support of two DB technologies [16] - LevelDB and CouchDB. LevelDB is the default and deals with the simple objects and the latter deals with JSON documents making it suitable for the complex objects and allows rich queries. The transaction log is nothing but blockchain which records all the transactions that result in the current values of the assets.

Membership Service Provider (or Certificate Authority): Membership Service Provider (MSP) is a component that aims to offer an abstraction of membership operation architecture. In particular, MSP abstracts away all cryptographic mechanisms and protocols behind issuing and validating certificates, and user authentication. Each organization will maintain their, own MSP.

Organization: Organizations logically separates members (peers) and the may or may not share the MSPs. It is recommended to have one MSP per organization and we follow that recommendation. In our case, ours stakeholders are the organization. 
Channel: A Hyperledger Fabric channel is a private "subnet" of communication between two or more specific network members, for the purpose of conducting private and confidential transactions. Each peer of every organization that joins a channel has its own identity given by a membership services provider (MSP), which authenticates each peer to its channel peers and services.

Smart contracts (chaincode): A smart contract is an agreement between two people in the form of computer code. They run on the blockchain, so they are stored on a decentralized database and cannot be changed. The transactions that happen in a smart contract processed by the blockchain, which means they can be sent automatically without a third party. So far there may be three smart contracts (not limited) in our blockchain system.

Assets: Hyperledger Fabric assets can range from intangibles like contracts and intellectual property to tangible like real estates or any goods. These assets can be modified by using special chaincode transactions. In Fabric, the assets are defined as a collection of key-value pairs with state changes recorded as transaction on the ledger.

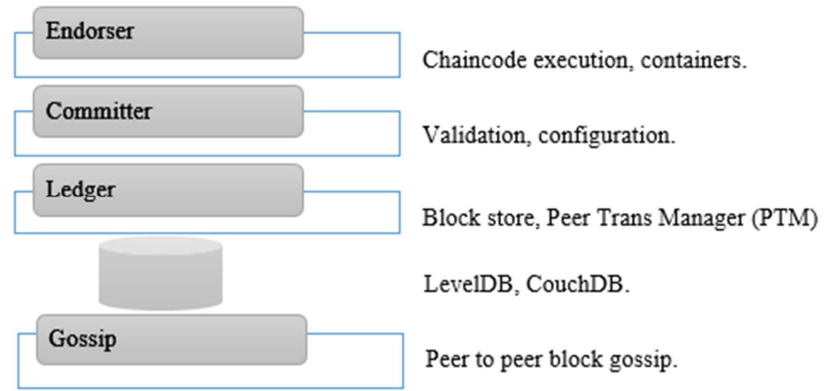

Source: (Elli Androulaki and Christian Cachin, 2018).

Figure 8. Components of Fabric.

\subsubsection{Transaction Flow}

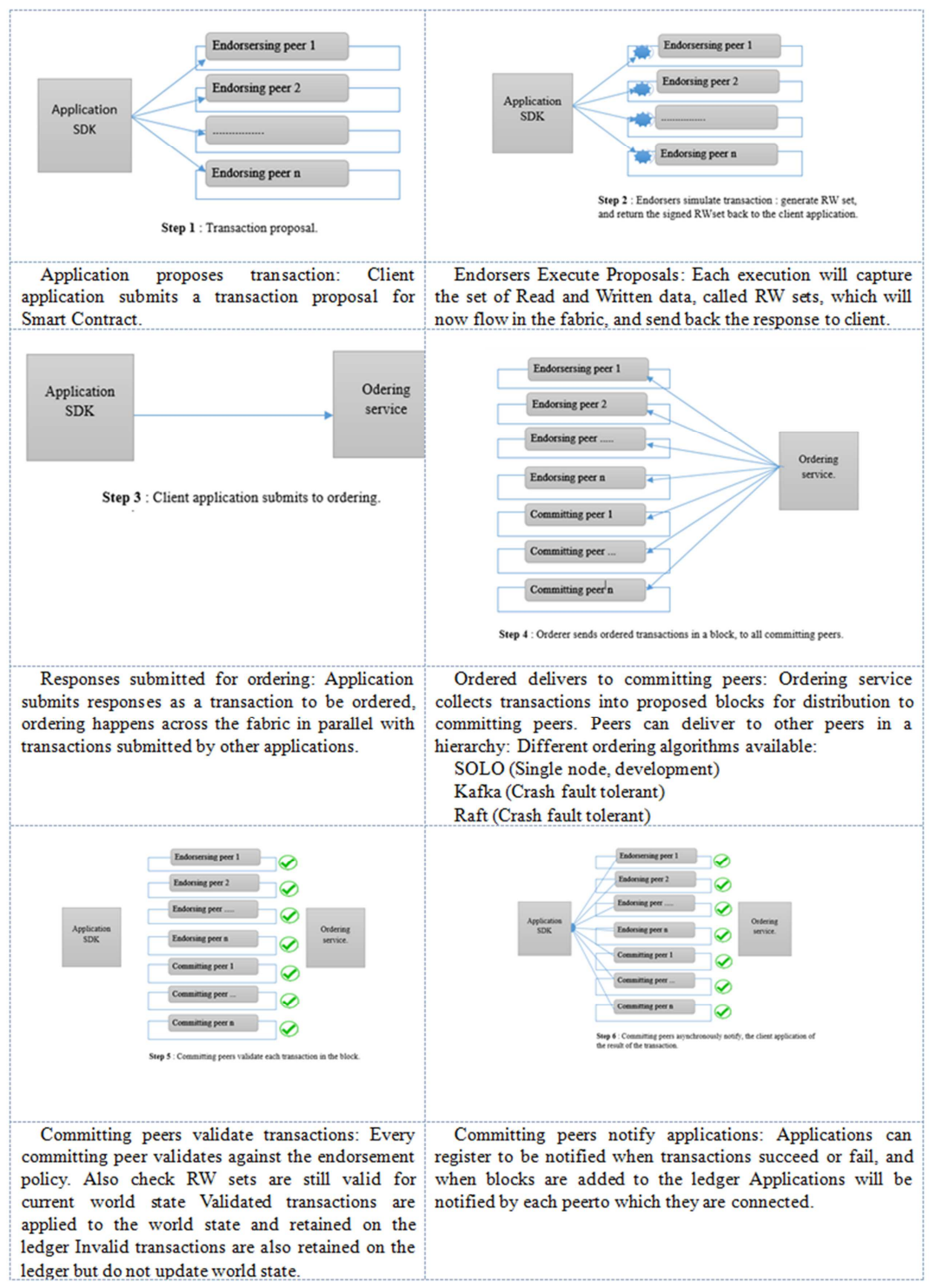

Figure 9. Transaction flow. 


\subsubsection{Case Study: Land Registry in Cameroon}

To implement, we will need the following structures for a start:

Ministry of Domains, Cadaster and Land Affairs: for the registration of land titles, and updates (mortgage, fragmentation land, donation, transfer, sale, etc.), represented on the figure below by MINISTER 1 .

1. An endorsing peer.

2. An ordering peer.

3. A committing peer.

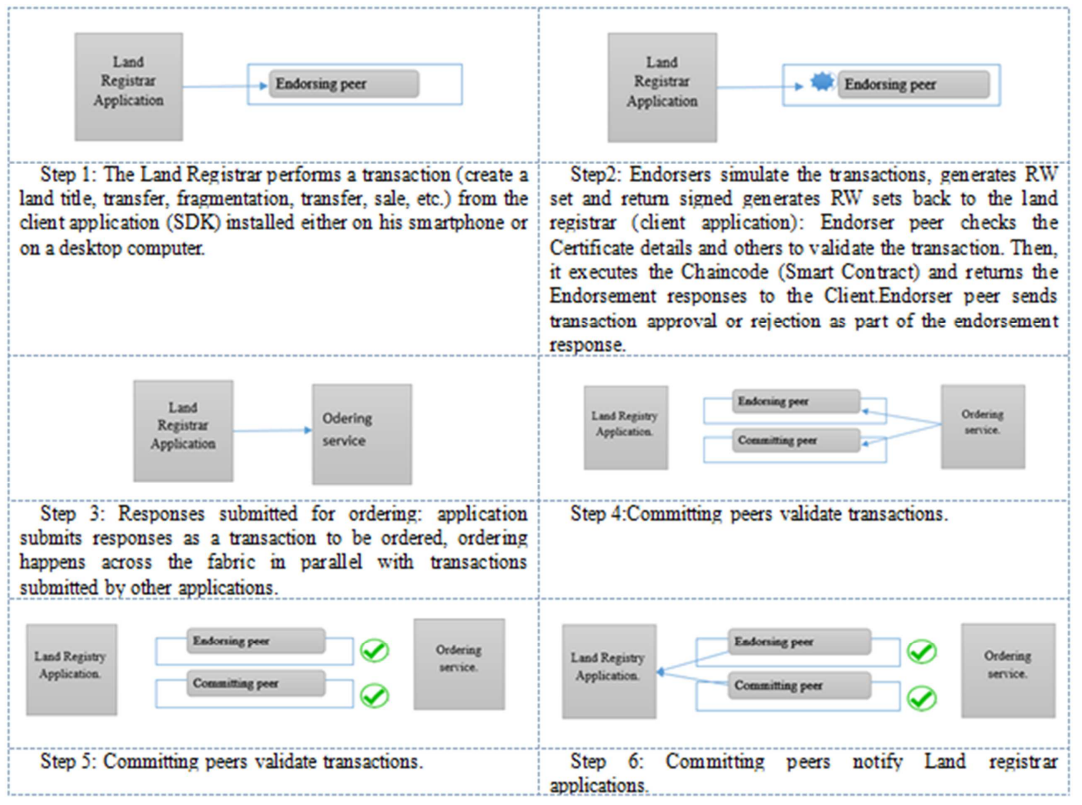

Figure 10. Sample Fabric network with two organizations.

\section{Scalability's Analysis and Improvement of the Development of the Hyperledger Fabric [15]}

On fabric network, we have different types of nodes with their own responsibilities, and allow them to configure a network of nodes to scale independent of each other. It's not possible in advance, to predict the number of peers in a network and the number of orderers. There is no parallel relationship between the number of endorsers and committers. Endorsers may have to execute heavy computations, but since endorsers are separated from the ordering service it does not affect it's execution time. Developers are therefore free to write more complex applications that are costly to execute without disrupting the ordering service or any other application on the network.

Endorsing peers must be powerful machines.

Perform tests by gradually adding Endorsing peers, in order to have the maximum number of Endorsing peers that we will need for our network.

In general, it's better to have a powerful peer endorser per channel.

\section{Result Analysis of Land System with the Blockchain and Discussion}

According to the previous methodology, we now can complete the issues discovers during the review study.

\begin{tabular}{|c|c|c|}
\hline Problems & $\begin{array}{l}\text { land registry } \\
\text { office's solutions }\end{array}$ & Weaknesses \\
\hline $\begin{array}{l}\text { Dilapidated } \\
\text { state of the } \\
\text { old Land } \\
\text { registers. }\end{array}$ & Transcription. & $\begin{array}{l}\text { Transcribe the information from the old land } \\
\text { books into the new land books in order to } \\
\text { standardize the information. }\end{array}$ \\
\hline
\end{tabular}

Restore the Land

Office fire. Title with the user's copy (duplicate).
If a fire happens to occur at the land office level, then the Land registrar would use the copies of users' Land Titles to restore the Land Titles from the Land Register. This does not always guarantee the reliability of the solution, because if the user had a fake, we will then assign an authentic title to a fake duplicate.

\section{Strengths: Hyperledger Fabric's solutions}

It's like information written on marble; as long as the blockchain exists the information exists.

The blockchain is like a large distributed register which is copied in several places, if we lose in one place, we end up in another. 


\begin{tabular}{|c|c|}
\hline $\begin{array}{l}\text { Verification of } \\
\text { the } \\
\text { authentication } \\
\text { of a Land } \\
\text { Title. }\end{array}$ & $\begin{array}{l}\text { Establishment of } \\
\text { property } \\
\text { certificates. }\end{array}$ \\
\hline $\begin{array}{l}\text { Loss of a } \\
\text { Land Title. }\end{array}$ & $\begin{array}{l}\text { Procedure for } \\
\text { establishing a } \\
\text { Duplicate. }\end{array}$ \\
\hline $\begin{array}{l}\text { Disappearance } \\
\text { of the sheets } \\
\text { (folios). }\end{array}$ & $\begin{array}{l}\text { Authorization of } \\
\text { judge to restore } \\
\text { lost sheet. }\end{array}$ \\
\hline $\begin{array}{l}\text { Sale of false } \\
\text { Land Titles or } \\
\text { double. sale. }\end{array}$ & $\begin{array}{l}\text { identify the } \\
\text { Perpetrators of } \\
\text { this criminal. act } \\
\text { and to bring } \\
\text { them to justice. }\end{array}$ \\
\hline $\begin{array}{l}\text { Disappearance } \\
\text { of the Land } \\
\text { Register, } \\
\text { register of } \\
\text { deposits, and } \\
\text { file. }\end{array}$ & $\begin{array}{l}\text { Restore the land } \\
\text { register with the } \\
\text { help of the } \\
\text { judge, and users } \\
\text { in possession of } \\
\text { their duplicates. }\end{array}$ \\
\hline
\end{tabular}

A land title is very often the subject of transactions: mortgage, sale, transfer, and many others. To verify this, simply establish a certificate of ownership to prove that a Land Title is free from all transactions at a given time.

If a user has lost his Land Title, there is a procedure for establishing a Duplicate.

The folio represents the sheet dedicated to the authentic information of a Land Title; if it disappears, we could take advantage to tamper with, tamper with the user's Land Title.

The sale of fake Land Titles.

Restore the Land Title with the user's copy, or restore the Land Book with the users' copies. In short, the Curator uses his intuition and the judge's help to establish the Land Titles by all the technical means in his possession.
The land register being accessible through a network, it is very easy to check the status of a Land Title of a user at a given time. So no need for a procedure to establish a certificate of ownership.

The land register being accessible through a network, no need for a Duplicate establishment procedure.

It is impossible for the sheet of the land registrar to disappear, because it's managed by a secure system.

It is impossible for one to find false land titles, indeed one of the characteristics of the blockchain is its security.

It's impossible to lose the land register, because of its distributed nature.

\section{Conclusion and Future Scope}

Blockchain is a very promising new technology. It solves several problems related to the storage of insecure data. In this paper we have presented a framework to improve the security and scalability of the land register in Cameroon. Development of the project continues. The next stage will be the development of smart contract transaction scripts and access control lists. Conduct several tests on virtual machines the next step will be the creation of a front-end architecture for easy access of users to the designed system. Others authors can ameliorate this Framework with the methods for geospatial.

\section{References}

[1] Ashwin Sekhari, Rishav Chatterjee, Ras Dwivedi. (2019). Entangled Blockchains in Land Registry Management. Information Security Research \& Development Center. https://isrdc.iitb.ac.in/blockchain/workshops/2019-iitb/papers/paper$22 \% 20$ Entangled $\% 20$ Blockchains $\% 20$ in $\% 20$ Land $\% 20$ Registry $\% 2$ 0Management.pdf.

[2] Tara Salman, Maede Zolanvari, Aiman Erbad. (2018). Security Services Using Blockchains: A State of the Art Survey. https://ieeexplore.ieee.org/abstract/document/8428402.

[3] D. Lee Kuo Chuen, Ed. (2015). Handbook of Digital Currency. EconPapers, https://econpapers.repec.org/RePEc:eee:monogr:97801280211 70.

[4] Z. Zheng, S. Sie, X Chen. (2017). An Overview of Blockchain
Technology: Architecture, Consensus, and Future Trends. IEEE international, https://ieeexplore.ieee.org/abstract/document/8029379/.

[5] V. Buterin. (2014) "A next-generation smart contract and decentralized application platform," white paper. Cryptorating.eu,

https://cryptorating.eu/whitepapers/Ethereum/Ethereum_white _paper.pdf.

[6] L. Lamport, R. Shostak, and M. Pease. (1982). The byzantine generals problem, ACM Transactions on Programming Languages and Systems (TOPLAS), vol. 4, no. 3, pp. 382-401.

[7] S. Nakamoto. (2008). Bitcoin: A peer-to-peer electronic cash system. Bitcoin.org, https://bitcoin.org/bitcoin.pdf.

[8] P. Vasin. (2014). Blackcoins proof-of-stake protocol v2. blackcoin.co.

[9] S. King and S. Nadal. (2012). Ppcoin: Peer-to-peer cryptocurrency with proof-ofstake.https://www.chainwhy.com/upload/default/20180619/12 6a057fef926dc286accb372da46955.pdf.

[10] C. Miguel and L. Barbara, "Practical byzantine fault tolerance," in Proceedings of the Third Symposium on Operating Systems Design and Implementation, vol. 99, New Orleans, USA, 1999, pp. 173-186.

[11] THE LINUX FONDATION. (2015). Hyperledger project. https://www.hyperledger.org/.

[12] S. R. Bryatov, A. A. Borodinov, Blockchain technology in the pharmaceutical supply chain: researching a business model based on Hyperledger Fabric.

[13] THE LINUX FONDATION. (2015). https://www.hyperledger.org/use/fabric. 
[14] E. Androulaki, C. Cachin. (2018). Hyperledger Fabric: A Distribute d Operating System for Permissioned Blockchains. ACM Digital library, https://dl.acm.org/doi/pdf/10.1145/3190508.3190538.

[15] M. Scherer. Performance and Scalability of Blockchain Networks and Smart Contracts. (2017). UMEA University,
https://umu.divaportal.org/smash/get/diva2:1111497/FULLTE XT01.pdf.

[16] T. T. A. Dinh, R. Liu, M. Zhang, G. Chen, B. C. Ooi, and J. Wang. (2017). Untangling blockchain: A data processing view of blockchain systems. IEEE, https://www.comp.nus.edu.sg/ ooibc/blockchainsurvey.pdf. 